\title{
Development of critical care nursing in Hunan,
}

\section{China}

Ze Ya Shi RN; CNS, PhD, Associate Professor, Deputy Director of Department of Nursing, People's Hospital of Hunan Province, Changsha, China.

Qun Wang RN; PhD, Postdoctoral Fellow. The Nethersole School of Nursing, The Chinese University of Hong Kong. Hong Kong

SAR, China.

Yue Lan Qin RN; MSN, Professor, Vice President, People's Hospital of Hunan Province, Changsha, China.

Yi Min Zhu, Chief Physician, PhD, President, People's Hospital of Hunan Province, Changsha, China.

Yan Hui Liu, RN; MSN, Department of Nursing, People's Hospital of Hunan Province, Changsha, China.

Yan Hong Wu, Attending Physician, ICU Department, People's Hospital of Hunan Province, Changsha, China.

Key Words: critical care nursing human resources $\quad$ management $\quad$ specialty development

E-mail: 912542420@qq.com

\section{SUMMARY}

- Critical care nursing (CCN) is an important area in health care system, which provides focused monitoring and intensive treatment to critically ill patients.

- In spite of a late start, the $\mathrm{CCN}$ in Hunan Province has achieved significant progress in development of intensive care units (ICUs), quality management, specialty training and research.

- There are some challenges for $\mathrm{CCN}$ development in Hunan Province, in terms of the quality management, nursing human resources, specialty training and research.

- Continuous improvements could be achieved by enhancing the ICUs in level-II hospitals, building a specialized nursing team, improving the management system, promoting nursing research, and encouraging international collaborations.

\section{INTRODUCTION}

The concept of critical care medicine (CCM) in China has developed since the early 1980s. With rapid social and economic developments, the Chinese has entered the stage of ageing and encountered great changes in the disease spectrum. Consistent with the emerging amount and severity of critical diseases, CCM has become a fundamental part and plays important roles in the Chinese health care system with the intensive care unit (ICU) becoming the key department in different hospitals (Guo et al., 2013). With the development of CCM, critical care nursing (CCN) has become a professional discipline, and the Chinese Nursing Association has established the special committee on CCN (Zheng et al., 2015).

The Hunan province is located in South Central China, covering an area of 211,800 square kilometers and with a population over $70,000,000$. Hunan is an important agricultural province, and is also famous for its rich mineral and travel resources. The province has achieved significant economic developments, and its urbanization has accelerated. In China, the hospitals are classified following a three-level system (level-I, -II, -III), in which the level-III stands for the highest level of hospitals. There are also three grades (grade-A, $-B,-C$ ) in each level, with the grade-A as the best in each level. To meet the increasing needs of critical care, some large III-A hospitals (such as the Xiangya Hospital and People's Hospital of Hunan
Province) have built the first few ICU departments, and have been providing focused monitoring and intensive care to patients all over the province. During the last two decades, level-II hospitals also have started to build their own ICU departments. Due to a late start and the high requirements in this discipline, there are many challenges to the development of $\mathrm{CCN}$ in Hunan, such as building a standardized management system and conducting higher quality of research. The aim of this paper is to review the development of $\mathrm{CCN}$ in Hunan and to promote its further development through learning the experience and advanced knowledge from international hospitals.

\section{THE DEVELOPMENT OF CRITICAL CARE NURSING IN HUNAN PROVINCE}

\section{History}

With limited understanding of critical care in the early stage, the critical patients were simply put in the wards or emergency rooms near the nurse station to facilitate close monitoring and care (Liu et al., 2013). Critical care was also understood as sending the postoperative patients to the recovery room to regain consciousness before moving them back to the general ward (Yu \& Tang, 2005; Yu \& Tang, 2007). In the early 1990s, the first groups of comprehensive ICU were established in a few large III-A hospitals following the CCM modes in western countries. Since then, with upgrades in facilities and equipment, the services started to achieve international standards. With the advancement of knowledge in CCM and CCN, a group of nurses specializing in $\mathrm{CCN}$ was established. After entering the 21st century, the discipline went into another rapid developmental stage when the former Ministry of Health required level-II or above hospitals to establish ICU. In the past decade, the number of beds and health care professionals in ICU departments has increased by 5 and 10 times, respectively. By October 2015, there were 141 ICU departments in Hunan province with 2,050 beds. Among the 2,843 nurses and nurse assistants (including 2,698 females and 145 males) working in ICU, there were 55 senior nurses, 361 middlelevel, 2,352 juniors, and 75 nurse assistants. In 2005, the Hunan Nursing Association established the special committee on $\mathrm{CCN}$ to standardize the management in ICU. Every year, the CCN special committee held training courses and academic communication workshops on critical care for ICU nurses, which enhanced the construction and continuous development of $\mathrm{CCN}$ in Hunan province (Zeng \& Huang, 2008). 


\section{Current ICU situation in Hunan Province}

In Hunan Province, CCM has faced an imbalanced development, resulting in a large gap in the requirements of setting up ICUs between level-II and level-III hospitals. The ICU departments in levelIII hospitals usually have a longer history, and thus their facilities, equipment, human resources and management could meet or even surpass the requirements of The Guideline of Discipline Construction and Management of Critical Care (GDCMCC) published by the National Health and Family Planning Commission (2009). These ICUs in level-III hospitals could provide continuous dynamic monitoring and treatment for critically ill patients, and deliver comprehensive functioning support to save patients' lives, e.g., support in respiration, circulation, and nutrition. Xiangya Hospital has an internationally compatible ICU, which is the most influential one in South China. Some level-III hospitals, e.g., Xiangya Second Hospital and People's Hospital of Hunan Province also have built their highlevel central and specialized ICUs. However, the development of ICUs in most level-II hospitals is not up to standard. Due to the limitations in facilities, equipment and human resources, the amount and quality of health care professionals in these ICUs could not meet the requirements of GDCMCC (Fan \& Feng, 2013).

In spite of the rapid development recently, the current ICUs still could not meet the clinical demands. Beds in ICUs only account for $1.48 \%$ of total beds in hospitals and are overloaded all the time. According to the national requirements for level-III hospitals, the ICU departments should account for $5 \%$ to $8 \%$ of the total beds in the hospital, with a utilization rate ranging from $65 \%$ to $75 \%$ (Office of the National Health and Family Planning Commission, 2011). If the utilization rate exceeds $80 \%$, the hospital will have to expand the scale to meet the demands (Office of the National Health and Family Planning Commission, 2011). The number of ICU beds in the United States accounts for $10 \%-15 \%$ of the total beds. Moreover, their utilization rate had increased by $4.5 \%$ during 2000 to 2005 (Halpern \& Pastores, 2010). The shortage of nurses, especially the lack of experienced nurses also limits the development of ICU. In Hunan Province, the bed-to-nurse ratio is 1: 1.38-2.1, which is far below the national requirement of 1:2.5-3 (Office of the National Health and Family Planning Commission, 2011). Moreover, only $14.7 \%$ of the ICU nurses have senior-or-middle-level qualifications. Among the nurses with a bachelor degree, $82.6 \%$ of them have worked in ICU for less than 5 years. The shortage in human resources significantly influences the education and research in CCN. Addressing the problems caused by limited working experiences of highly educated nurses has become an inevitable challenge for ICU management.

\section{Critical nursing scheduling mode}

The nursing scheduling model mainly follows the APN continuous shift mode (Yue et al., 2009), in which the shifting hours for A (am) starts from 07:30 to 15:00, P (pm) from 14:30 to 22:00, and N (night) from $21: 30$ to $08: 00$. It normally takes 30 minutes for completing the shift reports. There is a team leader for each shift, who is appointed by the head nurse, working together with other nurses matched by their qualification levels and working experiences. Nurses are divided into small groups to take care of the patients. Requirements for the team leaders include, but not limit to: (1) with an associate degree or above; (2) with middle-level qualification level or above; (3) with more than 8-year working experience in ICU; and (4) with high responsibility, strong practical and coordination skills. They are responsible for the communication and coordination, supervising and guiding the nursing practice, as well as the quality control in the shift. The team leaders also arrange working duties of other nurses in the team according to their capacity. In general, junior nurses take care of the relatively stable patient and middle-level nurses take charge of the critically ill patients. The senior nurses work on relatively unstable patients and assist solving difficult nursing problems. In special occasions, the scheduling is flexible. For example, if there are unoccupied beds, the team leader could arrange some nurses to take leave according to manpower needs of the shift. There are detailed scheme for different levels of nurses, in terms of entry standards, position responsibilities, training requirements, assessment criteria, and salary scales. This scheme reflects the values of nurses in different levels, so that nurses follow the nursing process and provide 24-hour comprehensive monitoring and intensive treatment to the critical patients.

The APN continuous shift mode and hierarchy-based management can improve the role functioning and working passion of high-level nurses. Based on the effective communications and encouragement among nurses from different levels, patients' safety and well-being can be ensured, and the equivalence, fairness and safety of the nursing care can be well-established. This mode is well received by nurses, since they are able to arrange the work effectively under this scheduling mode. After all, this scheduling mode still needs further optimization. Till now, there is no unified standard or regulations for the hierarchy-based management mode in Hunan Province. The assessment system and salary scales also need to be enhanced to attract nurses to work in ICUs.

\section{Quality control}

To ensure the safety and quality of medical treatment in ICU, and to reduce treatment failures, the Health and Family Planning Commission of Hunan Province (2013) developed the "Quality Control and Assessment Standard of ICU" and the "Sensitive Parameters for Monitoring Nursing Quality in Hunan Province (Trial)". These guidelines were developed based on the current situations in Hunan Province, the international standard guidelines for critical care, and the global trends of ICU development. These guidelines provide clear descriptions and detailed evaluation methods for the disciplinary constructions in level-II and level-III hospitals, medical management, classification of admitted patients, requirements of monitoring techniques and facilities, as well as infection control measures. The generic parameters for nursing quality include the availability of first-aid equipment and medications (\%), risk assessment of fall/drop for hospitalized patients (\%), risk assessment of pressure ulcer (\%), incidence of fall/drop (\%), and incidence of fall/ drop-related injury $(\%)$, etc. Specific parameters for nursing quality in ICU include unplanned endotracheal extubation rate, ventilatorassociated pneumonia (VAP) rate, and incidence of central venous line related infection.

The head nurse announces the outcomes of sensitive parameters every month. The nurses are required to analyze the outcomes, identify the problems, and propose solutions. Modifications are implemented in the next month to reach the continuous quality improvement. Moreover, advanced nursing management approaches are applied to ensure the safety of nursing practices, e.g., evidencebased nursing practice, cooperation between physicians and nurses, Situation - Background - Assessment - Recommendation communication mode, and quality control circle.

There has been substantial improvement in the quality of critical care in Hunan Province. However, the quality control in CCN, especially the safety management, has to deal with various challenges, which are mainly caused by the shortage in human resources, limited skills and experiences among current nurses, and the slow development in management regulations and monitoring mechanism (Zhao et al., 2013).

\section{Specialty training for ICU nurses}

According to the standards of nursing specialization, one of the objectives of nursing development is to train specialized ICU nurses to meet the requirements of modern nursing practice and the 
needs of the critically-ill patients. In Hunan Province, the training of specialized ICU nurses was initiated since 2008 under a jointhospital-training mode. According to the joint assessment of the Quality Control Center for Specialized Nursing under the Department of Health in Hunan Province and the Nursing Association in Hunan Province, Xiangya Hospital of Central South University, the Xiangya Second Hospital, the Xiangya Third Hospital, People's Hospital of Hunan Province, and the Center Hospital of Changsha City were appointed as qualified hospitals to provide specialized trainings for ICU nurses. The Specialized Intensive Nursing Care was employed as the textbook. The provincial-wide training is open to all ICU nurses. Hospitals select potential nurses to attend this three-month full-time training course, which consists of a one-month theoretical training and two-month practical training. Participants are required to pass the theoretical and practical evaluation before being issued a certificate by the Quality Control Center in Hunan Province. So far, there have been 385 specialized ICU nurses in Hunan Province. In addition, 24 nurses had participated in the specialty training organized by the Chinese Nursing Association, and 523 nurses took part in the training programs organized by advanced ICUs in Beijing and Guangzhou for at least six months. With the leading of specialized nurses, the quality of critical nursing has been improved significantly, indicating the importance of ICU specialization (Zeng \& Tang, 2008).

Due to the late start in specialized ICU nurses, qualification authentication and standardized training are still under exploration. The number of nurses attending these trainings is far from the clinical demands, and a gap still exists between the quality of the training and clinical expectation. Until now, the attendance of training has not been included in requirements for employment, promotion and payment, which leads to the waste of human resources and diminishes the working passion of specialty nurses. Furthermore, the actual training time cannot really meet the requirement, which may be ascribed to the shortage of nurses and difficulties in attending fulltime training (Huang et al., 2015).

\section{Research}

CCN has been a commonly identified area in nursing research. Recently, ICU nurses in Hunan Province have conducted series of research based on the clinical needs and identified clinical problems. The research focused on ICU human resource management (Zhou et al., 2015), training and usage of specialized ICU nurses (Zeng \&Tang, 2008; Zhao et al., 2013), sedation assessment and nursing (Liu et al., 2015), prevention and management of multidrugresistant organism infection (Huang et al., 2015; Zheng et al., 2014), management of nasal-intestinal tube and enteral nutrition (Liu et al., 2015), glycemic control among ICU patients (Shi et al., 2013; Shi et al., 2014a), safety management and quality control (Cao et al., 2010a; Shi et al., 2014b), management of respiratory and circulatory support (Cao et al., 2010b; Wei, 2012), end-of-life care (Li \& Ouyang, 2002), as well as lying position for rehabilitation (Peng et al., 2012). At the same time, referring to the cluster bundled nursing strategy from other countries, a multi-centered research platform for CCN was established by Hunan Critical Nursing Association. The platform employs evidence-based nursing to guide the development and application of standardized ICU practice, which will benefit the patients at large (Li \& Zhao, 2011).

However, due to the late development of $\mathrm{CCN}$, the lack of researchers, heavy workload of nurses, and limited funding support, the outputs of ICU nursing research in Hunan are still low.

\section{FUTURE DEVELOPMENT OF CCN IN HUNAN PROVINCE}

During the last two decades, the development of $\mathrm{CCN}$ in Hunan Province has accelerated. The ICUs have achieved significant progress in the construction and management, professional education, nursing research and practice. However, when compared to the ICUs in other advanced areas, the ICU development in Hunan still has some shortcomings. Currently, the CCN aims at gradual specialization through specialized education and continuous improvements in nursing quality. The goals and opportunities for future development are discussed as following.

\section{Enhancing the ICU development in level-II hospitals}

ICU in level-II hospitals is an important part of the national health care, which is also one of the limitations in CCN development in Hunan Province. To keep the balanced ICU development between level-II and level-III hospitals, improving the quantity and quality of the nurses should be firstly implemented. On one hand, increasing the number of nurses could raise the bed-to-nurse ratio to meet the national standard. On the other hand, part-time training could enhance the capacity of nurses. Secondly, many hardwares can be upgraded, including increasing the financial input, improving the environment, and purchasing/ upgrading necessary equipment and facilities. Thirdly, improving the management and service quality could ensure the safety of the patients. Nevertheless, close and extensive connections should be built between hospitals of different levels. Effective communications and training programs between hospitals could ensure a synchronous development (Qiu, 2013).

\section{Building a specialized team of ICU nurses}

The Nursing Association in Hunan Province can play several roles to build a specialized team of ICU nurses. Experiences and resources can be sought from other regions that have already set up successful ICU, such as Beijing and Hong Kong. Special funds should be made available for such development in this area. To accelerate the speed of specialization, certificates of ICU trainings should be required. Both existing nurses and novices should attend trainings as entry requirement of working in ICUs. For those nurses who have already undergone the specialty training, priorities in duty arrangement, promotion, and salary increase should be offered. Hopefully, the appropriate use of human resources could motivate nurses' working passion and excavate their potential and abilities. The hospitals should also contribute to building a specialized team of ICU nurses through following the national requirements and considering their own actual situations (Zeng \& Tang, 2008).

\section{Improving the management system of $\mathrm{CCN}$}

According to the requirement of GDCMCC, a comprehensive management system for $\mathrm{CCN}$ should be built to ensure the continuous improvements in the quality of critical care. Firstly, the practice standards, position description, and management rules should be further developed. Secondly, the patient-centered nursing work flow should be optimized and the basic physical needs of the patients must be satisfied. Thirdly, the informatics in ICU should be built, which will contribute to data-based management. In addition, patients' safety should always be regarded as the core task. An early warning mechanism should be established to improve the response speed in emergencies (Cao et al., 2010a; Ren, 2012).

\section{Promoting research}

The nurturing of researchers in $\mathrm{CCN}$ needs to be strengthened. The research should keep pace with the trends in international research, such as the risk assessment and emergency response mechanism, bundle care, palliative care, goal-oriented monitoring, patients' comfort, inpatient infection control, and prevention of complications. With help of the multi-centered research platform, research data could be collected from different centers. The implementation of evidence-based nursing research and patient-outcome-centered studies would improve the $\mathrm{CCN}$ research levels in Hunan Province. 


\section{Encouraging international collaborations}

Encouraging international collaborations is one of the solutions to promote the development of $\mathrm{CCN}$. International collaborations could provide valuable experiences and advanced knowledge in $\mathrm{CCN}$ development, so that some pitfalls could be prevented and the development could be accelerated. Moreover, international training programs could be established to build a group of nurses following the international standard (DeJong et al., 2013; DeMul et al., 2012).

\section{CONCLUSION}

In conclusion, the $\mathrm{CCN}$ in Hunan Province has achieved significant progress in a short period of development. There are also many challenges and opportunities in human resources, management, and research. It is believed that the $\mathrm{CCN}$ development will reach to a higher level in the future.

\section{REFERENCES}

Cao L, Tang CX, Jiang DM (2010a). Application of "Ten Safety Goals" for patient in ICU. Chinese Nursing Research 24(19), 1764-1765. [Chinese].

Cao L, Tang CX (2010b). The application of PiCCO in monitoring one patient after lung transplant: a case report. Journal of Nurses Training 25(11), 1024-1025. [Chinese].

DeJong A, Molinari N, Terzi N et al (2013). Early identification of patients at risk for difficult intubation in the intensive care unit: development and validation of the MACOCHA score in a multicenter cohort study. American Journal of Respiratory and Critical Care Medicine 187(8), 832-839.

DeMul M, Alons P, Van der Velde P, Konings I, Bakker J, Hazelzet, J (2012). Development of a clinical data warehouse from an intensive care clinical information system. Computer methods and programs in biomedicine 105(1), 22-30.

Fan XR, Feng H (2013). The analysis of current situations and continuous improvements in the departments of critical care in level II hospitals. Chinese General Practice Nursing 11(7), 646-647. [Chinese].

Guo FM, Yang Y, Qu HB (2013). The development of critical care medicine in the past 10 years. Chinese Journal of Internal Medicine 52(2), 130-132. [Chinese].

Halpern NA, Pastores SM (2010). Critical care medicine in the United States 2000-2005: An analysis of bed numbers, occupancy rates, payer mix, and costs. Critical Care Medicine 38(1), 65-71.

Health and Family Planning Commission of Hunan Province (2013) developed the Investigation of Sensitive Parameters for Quality Control in ICU departments of Level III Hospitals. [online]. Available at: http:// www.hunanwst.gov.cn/ywpd/jkcj/201307/t20130716_13291.html. Accessed on $10 \mathrm{Feb}$ 2016. [Chinese].

Huang J, Zhang Y, Li LZ, Zhao LP, Chen YY (2015). Problems and commences on current status of Specialist Nurses training management in China. Chinese Nursing Management (2), 243-245. [Chinese].

Huang X, Deng ZD, Ni YX et al (2015). Chinese experts' consensus on prevention and control of multidrug resistance organism healthcareassociated infection. Chinese Journal of Infection Control (1), 1-9. [Chinese].

Li CH, Zhao XM (2011). Care of personalized humidification of artificial airway. Chinese Medicine Modern Distance Education of China 9(7), 67-69. [Chinese].

Li YL, Ouyang YY (2002). Strengthening nursing in terminal care. Chinese Journal of Hospital Administration 18(11), 678-679. [Chinese].

Liu L, Liu DW, Qiu HB (2013). Critical care medicine in China: history and perspective. Chinese Medical Journal, 126(10), 1806-1808. [Chinese].

Liu YH, Zhang JD, Shi ZY (2015). The application of blinding insertion of naso-jejunum feeding tube among surgical critical patients. Journal of Clinical Research 32(12),2302-2303. [Chinese].

Liu WJ, Liu MH, Luo TN (2015). Clinical effect of sedation care in pediatric patients using ventilator. China Medical \& Engineering 22(1),97-98.
[Chinese].

Office of the National Health and Family Planning Commission of the People's Republic of China (2009). The Guideline of Discipline Construction and Management of Critical Care. Report of the National Health and Family Planning Commission of the People's Republic of China [online]. Available at: http://www.moh.gov.cn/mohyzs/ s3577/200902/39165.shtml. Accessed on 10 Feb 2016. [Chinese]

Office of the National Health and Family Planning Commission of the People's Republic of China (2011). The Assessment Criteria and Rules for Level III Hospitals (the 2011 version). Report of the National Health and Family Planning Commission of the People's Republic of China [online]. Available at: http://www.nhfpc.gov.cn/yzygj/s3585u/201112/06f 754a213d8413787904e9e6439d88b.shtml. Accessed on 10 Feb 2016. [Chinese].

Peng XB, Tang CX, Li YL (2012). Effects and Nursing for Modified Prone Position Ventilation in Aged Patients with Pulmonary Complications after Abdominal Surgery. Nursing Journal of Chinese People's Liberation Army 29(9), 71-73. [Chinese].

Qiu XW (2013). Analysis of the situation and thinking about the bottleneck period of general ICU development in grass-root hospital. Modern Hospital 13(12), 96-98. [Chinese].

Ren YH (2012). New perspectives for nursing quality management of surgical ICU departments in general hospitals. Chinese Journal of Misdiagnostics 12(16), 4117-4118. [Chinese].

Shi ZY, Tang SY, Chen YX, et al (2013). Prevalence of stress hyperglycemia among hepatopancreatobiliary postoperative patients. International Journal of Clinical and Experimental Medicine 6(9),799803.

Shi ZY, Tang SY, Chen YX, Lee DT, Chair SY, Jiang B, Zhu X, Pan XJ, Yang JX, Qin YL (2014a). Application of a glycaemic control optimization programme in patients with stress hyperglycaemia. British Association of Critical Care Nurses, [Article first published online: 28 Oct 2014]. DOI: 10.1111/nicc.12121.

Shi ZY, Yang D, Qin YL et al (2014b). Effect of Quality Control Circle on Reducing Incidence of Restraint Defects of ICU Patients. Journal of Nursing (15), 13-16. [Chinese].

Wei HY (2012). The application of mechanical ventilation in emergency treatment of pediatric patients with severe asthma. Heilongjiang Medicine Journal 25(5), 758-760. [Chinese].

Yu YH, Tang CX (2005). Observation and disposal for the elderly patient in general anesthesia convalescence. China Medical \& Engineering 13(5), 553-554,557. [Chinese].

Yu YH, Tang CX (2007). Notions and methods of nursing management in post-anesthesia care unit. Journal of Nursing Science 22(12),11-12. [Chinese].

Yue LQ, Li YL, Gao HM (2009). Implementing the continuous shift scheduling mode to reduce the legal risks for nurses. Chinese Nursing Management 9(12), 63-65. [Chinese].

Zeng Q, Tang SY (2008). Perspectives on the development of specialty nurse in Hunan Province. Today Nurse (5), 29. [Chinese].

Zeng Q, Huang J (2008). Notions and Methods of Nursing Management in Post-anesthesia Care Unit. Journal of Nursing Science 8(9), 15-17. [Chinese].

Zhao LP, Li LZ, Huang J, Fan WJ, Chen YY, Liu J (2013). Study on influence of medical quality and medical safety SAFE-CARE system on inpatients' satisfaction. Chinese Nursing Research 27(22), 2381-2383. [Chinese].

Zheng XL, Quan MT, Wei QM, Wu YQ, Wang WL, Zhang YM, Li Y, Hu RJ, Pan HP (2015). Research of Delphi method on building nurse professional quality objectives of "Emergency Nursing". Chinese Journal of Medical Education 35(2),212-215. [Chinese].

Zheng Y, Liu MH, Bi Y, Fu XQ (2014). The influences of adherence to hand hygiene on infection in hospitals. Today Nurse (9),155-157.

Zhou YH, Li LZ, Xie X, Dai AP (2015). The applicability of three kinds of scoring systems in the prediction of nursing manpower needs in intensive care units. Chinese Journal of Nursing 50(5), 570-573. 